Supporting Information

\title{
Regular Two-Dimensional Molecular Array of Photoluminescent Anderson-type Polyoxometalate Constructed by Langmuir-Blodgett Technique
}

Takeru Ito, ${ }^{\dagger}$ Hisashi Yashiro, ${ }^{\ddagger}$ and Toshihiro Yamase ${ }^{* \dagger, \$}$

Chemical Resources Laboratory, Tokyo Institute of Technology, R1-21, 4259 Nagatsuta, Midori-ku, Yokohama 226-8503, Japan, Application Laboratory, Rigaku Corporation, 3-9-12 Matsubara-cho, Akishima-shi, Tokyo 196-8666, Japan, and Core Research for Evolutional Science and Technology (CREST), Japan Science and Technology Agency (JST), 4-1-8 Honcho, Kawaguchi 332-0012, Japan

\footnotetext{
* To whom correspondence should be addressed. E-mail: tyamase@res.titech.ac.jp.

${ }^{\dagger}$ Tokyo Institute of Technology.

* Rigaku Corporation.

$\S$ Japan Science and Technology Agency.
} 


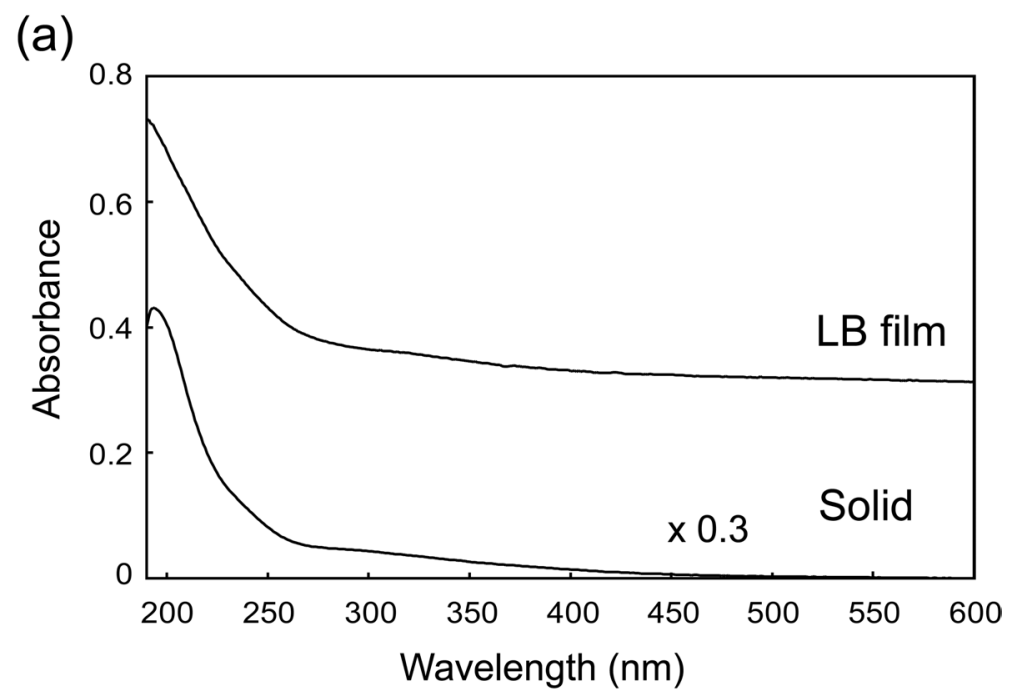

(b)

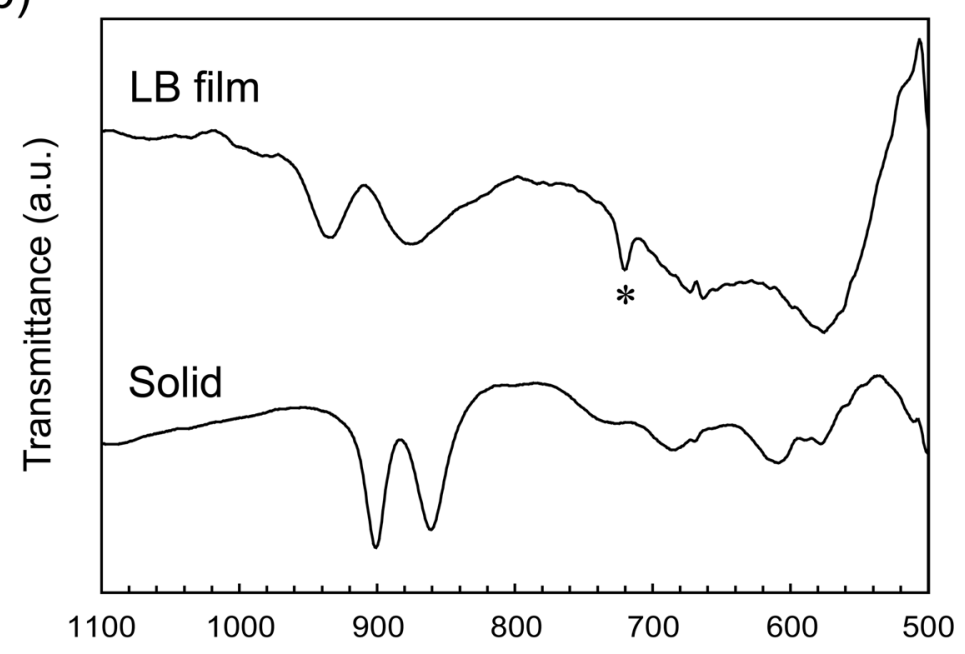

Figure S1. (a) UV-vis spectra of $\mathrm{MnW}_{6} / \mathrm{DODA} \mathrm{LB}$ film (29 layers) and $\mathrm{K} \cdot \mathrm{Na} \cdot \mathrm{MnW}_{6}$

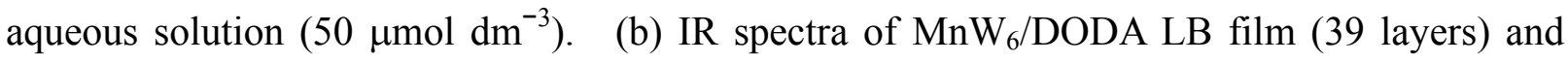
$\mathrm{K} \cdot \mathrm{Na} \bullet \mathrm{MnW}_{6}$ solid ( $\mathrm{KBr}$ pellet). A peak indicated by asterisk was derived from DODA cation. 


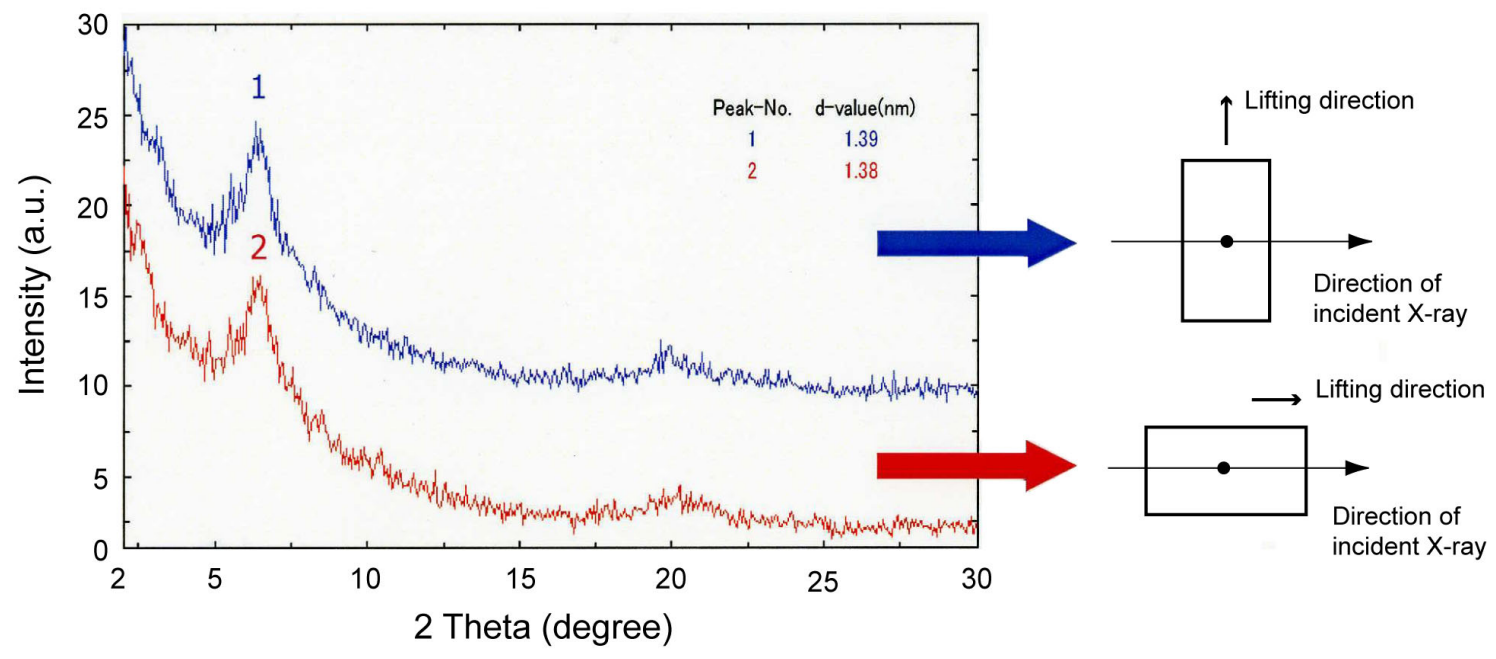

Figure S2. GIXD profiles of $\mathrm{SbW}_{6} / \mathrm{DODA} \mathrm{LB}$ film (25 layers) measured by changing angle between the direction of incident X-ray beam and LB film substrate $\left(90^{\circ}\right.$ and $\left.0^{\circ}\right)$. The upper profile is the same as that shown in Figure 6a.
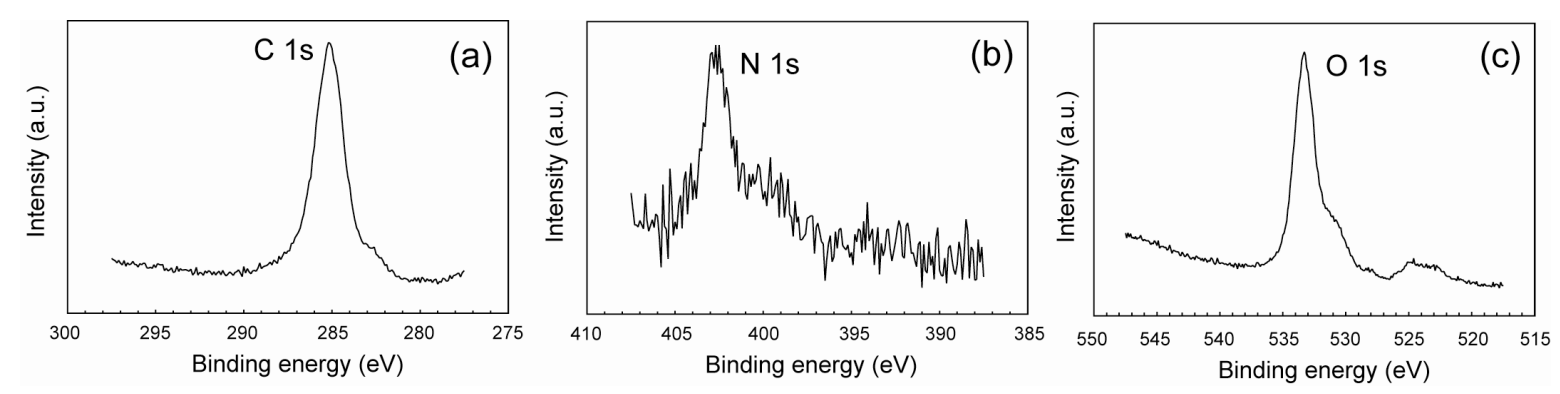

Figure S3. XPS spectra of $\mathrm{SbW}_{6} / \mathrm{DODA} L B$ film (21 layers): (a) $\mathrm{C} 1 \mathrm{~s}$, (b) $\mathrm{N} 1 \mathrm{~s}$, (c) $\mathrm{O} 1 \mathrm{~s}$ region. 